\title{
Ultrafast X-Ray Spectroscopy for Structural Dynamics Studies in Chemistry and Biology
}

\author{
W. Gawelda ${ }^{1,2}$, V. T. Pham ${ }^{1}$, A. El Nahhas ${ }^{1}$, S. L. Johnson ${ }^{2}$, D. Grolimund ${ }^{2}$, \\ M. Kaiser ${ }^{2}$, R. Abela ${ }^{2}$, M. Chergui ${ }^{1}$, C. Bressler ${ }^{1}$ \\ ${ }^{1}$ Laboratoire de Spectroscopie Ultrarapide (LSU), Ecole Polytechnique Fédérale de Lausanne (EPFL), BSP, \\ CH-1015 Lausanne, Switzerland \\ ${ }^{2}$ Swiss Light Source, Paul Scherrer Institut, CH-5232 Villigen-PSI, Switzerland
}

\begin{abstract}
Time-resolved x-ray absorption fine structure (XAFS) spectroscopy with picosecond temporal resolution is a new method to observe electronic and geometric structures of short-lived reaction intermediates. It combines an intense femtosecond laser source synchronized to the x-ray pulses delivered into the microXAS beamline of the Swiss Light Source (SLS). We present key experiments on charge transfer reactions as well as spin-crossover processes in coordination chemistry compounds next to solvation dynamics studies of photogenerated atomic radicals.
\end{abstract}

Keywords: X-ray absorption spectroscopy, picosecond time resolution, spin-crossover, transition metal compounds, aqueous radicals.

PACS: 61.10.Ht, 61.20.Lc, 72.80.Ga, 75.30.Wx, 82.53.-k, 78.70.Dm, 82.20.-w, 82.20.Kh, 82.50.-m

\section{INTRODUCTION}

In photochemistry and photobiology the ultrafast photoinduced redistribution of charges in the valence orbitals leads to the forming and breaking of bonds, which eventually initiates chemical activity and biological functions.

In order to deepen our understanding of the underlying elementary steps of chemical reactivity, one would ideally wish to obtain simultaneous information about the electronic and the geometric structure of the molecule in real-time. Femtosecond laser spectroscopy has matured to a powerful method to investigate the electronic structure of reacting molecules, but without providing detailed information about the positions of the nuclei during the evolving reaction. On the other hand, static geometric structures are readily obtained exploiting $\mathrm{x}$-ray tools, such as $\mathrm{x}$ ray crystallography, diffraction and $\mathrm{x}$-ray absorption spectroscopy. The latter has also proven its utility to obtain complementary information about the electronic structure around the x-ray absorbing element as well. Combining ultrafast laser with $\mathrm{x}$-ray spectroscopies in a pump-probe scheme is thus a straightforward extension to investigate the elementary steps of chemical reactivity [1].

\section{X-Ray Absorption Spectroscopy}

An x-ray absorption spectrum from inner-shell electrons consists of a series of bound-bound transitions (below the ionization potential IP of the inner-shell electron), followed by the absorption edge jump itself. Right above the absorption edge one observes a complicated modulation of the absorption cross section due to multiple scattering of the ionized photoelectron by its nearest neighbors in the molecule. This spectral range is also called the XANES region (x-ray absorption near-edge structure), and it contains rich information about both the electronic (below IP) and the geometric structure (above IP) including bond angles and distances. From about $50 \mathrm{eV}$ above IP to higher energies mainly single scattering events dominate, which result in a weak oscillatory modulation of the absorption cross section. This region is called the EXAFS region (extended $\mathrm{x}$-ray absorption fine structure), and delivers precise information about the internuclear distance of the nearest neighbors from the absorbing central atom. Thus one can obtain in one single spectrum simultaneous information about the valence electronic structure next to the nuclear arrangement of the atoms in the molecular system. In 
addition, x-ray absorption fine structure spectroscopy (XAFS) delivers this information even in disordered systems, including solvated chromophores in liquids. For coordination compounds including transition metallic reaction centers in proteins, XAFS delivers unique information about the metal $d$-orbitals, which are usually forbidden using optical light. Extending the pump-probe scheme towards XAFS detection would thus permit us to gain entirely new insight into the details of chemical reactivity.

In this paper we describe the experimental details of this new setup, which has been successfully applied to study the short-lived excited states of photoexcited molecules.

\section{EXPERIMENTAL SETUP}

The experimental setup has been described previously [2-4]. Briefly, x-rays from a $\mathrm{Si}(111)$ monochromator enter the experimental hutch, where they are focused to ca. $50 \mu \mathrm{m}$ diameter onto the sample with a pair of KirkPatrick Baez (KB) mirrors (Fig. 1). X-ray signals are detected with 4 large-area avalanche photodiodes (APD), one each for transmission $\left(\mathrm{I}_{1}\right)$ and for the incident signal $\left(\mathrm{I}_{0}\right)$ scattered off a thin $\mathrm{Cr}$ foil, and two fluorescence APDs $\left(\mathrm{I}_{\mathrm{F} 1}, \mathrm{I}_{\mathrm{F} 2}\right)$ for $\mathrm{x}$-ray fluorescence from the sample. The sample consists of a free-flowing liquid jet, which is excited with $400 \mathrm{~nm}$ light from an amplified fs laser system. Spatial overlap including a measurement of the spot sizes on the sample between both laser and $\mathrm{x}$ ray beams is set via steering the laser beam onto the $\mathrm{x}$ ray spot on sample and monitored with an imaging CCD camera.

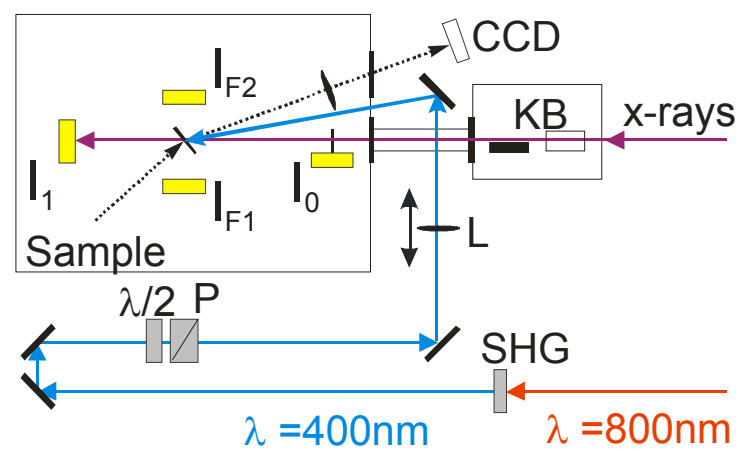

FIGURE 1. Experimental endstation with focused and overlapping laser and x-ray beams on the liquid sample. 4 detectors $\left(\mathrm{I}_{0}, \mathrm{I}_{1}, \mathrm{I}_{\mathrm{F} 1}, \mathrm{I}_{\mathrm{F} 2}\right)$ are used for measuring the XAFS, and an imaging CCD detector monitors spatial overlap and beam sizes. $800 \mathrm{~nm}$ light is frequency-doubled (SHG) and its pulse energy adjusted with a $\lambda / 2$ waveplate and polarizer $(\mathrm{P})$ combination before being focused (with the lens L) onto the sample. The x-ray beam is focused to ca. $50 \mu \mathrm{m}$ diameter with the KB optics.
We exploit a special electron bunch filling pattern at SLS for recording laser-pump-x-ray probe spectra (Fig. 2). The storage ring consists of 480 buckets, each separated by $2 \mathrm{~ns}$, in which 390 are filled (a filled bucket is also called an electron bunch) with ca. $6 \mathrm{x}$ $10^{9}$ electrons (thus generating each an average current of ca. $0.9 \mathrm{~mA}$ ), while in the subsequent $180 \mathrm{~ns}$ dark section a single densely packed (with up to 5 times more charge) hybrid electron bunch is placed. The $\mathrm{x}$ ray pulse from this electron bunch is used to probe the photoinduced changes of the x-ray absorption inside the sample.

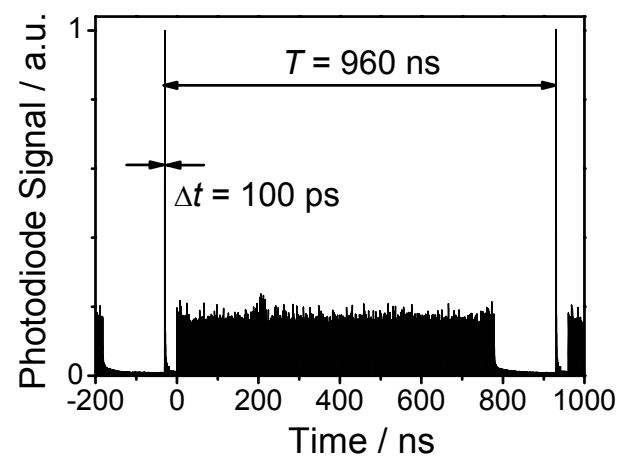

FIGURE 2. Bunch filling pattern at SLS (round trip time $960 \mathrm{~ns}$ ). Trains of 390 electron bunches with $2 \mathrm{~ns}$ spacing are followed by a $180 \mathrm{~ns}$ long empty section, in which a densely packed electron bunch (pulse width $100 \mathrm{ps)}$ ) is placed $30 \mathrm{~ns}$ before the start of the bunch train.

The amplified laser system for photoexcitation delivers $0.4 \mathrm{~mJ}, 400 \mathrm{~nm}, 120 \mathrm{fs}$ laser pulses at $1 \mathrm{kHz}$ repetition rate, which is synchronized to the round trip frequency $(T=960 \mathrm{~ns})$, thus ensuring a constant time delay between the laser and selected probing $\mathrm{x}$-ray pulses. A gated integrator delivers a sequence of output signals proportional to the input signal integrated over a fixed time window (Fig. 3). These signals are recorded with an ADC card, triggered at 4 $\mathrm{kHz}$, in order to provide a sequence of the x-ray signals when the laser is on and off, but also for recording the electronic background signal with no $\mathrm{x}$ rays present. First, the $\mathrm{x}$-ray signal at time $\Delta t$ after laser excitation is measured $\left(\mathrm{I}_{\mathrm{p}}\right)$, then a background signal without $\mathrm{x}$-rays is recorded after ca. $250 \mu \mathrm{s}$ $\left(\mathrm{I}_{\mathrm{bck}}(250 \mu \mathrm{s})\right)$, which enables post-correcting baseline drifts in the gated integrator, and finally, after $0.5 \mathrm{~ms}$, the x-ray signal from a fresh sample without laser excitation is recorded $\left(I_{u}\right)$ followed by a baseline correction measurement $\left(\mathrm{I}_{\mathrm{bck}}(500 \mu \mathrm{s}+250 \mu \mathrm{s})\right.$, not shown in the figure). The pump-probe signal in transmission is defined as the transient absorption $T_{\text {tr }}$ of the photoexcited sample via 


$$
T_{t r}(E, \Delta t)=\ln \left(\frac{I_{p u m}}{I_{u n p}}\right)
$$

with $I_{\text {pum }}=I_{\mathrm{p}}(\Delta t)-I_{\mathrm{bck}}(\Delta t+250 \mu \mathrm{s})$ and $I_{\mathrm{unp}}=$ $I_{\mathrm{u}}(500 \mu \mathrm{s})-I_{\mathrm{bck}}(500 \mu \mathrm{s}+250 \mu \mathrm{s})$ being both the baseline $\left(I_{\mathrm{bck}}\right)$ corrected photoexcited and unexcited $\mathrm{x}$ ray signals, respectively (see Fig. 3).

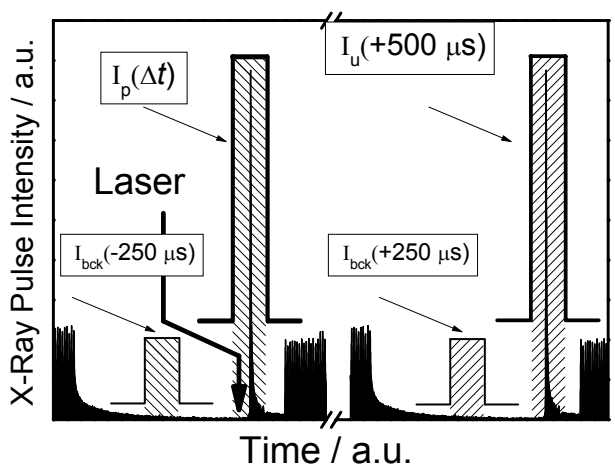

FIGURE 3. Data acquisition scheme employed at the microXAS beamline of the Swiss Light Source. A gated integrator measures sequentially the $\mathrm{x}$-ray intensity at the chosen time delay $\Delta t$, a background signal ca. $250 \mu$ s earlier and later, the x-ray signal of the unexcited sample after 500 $\mu$ s (each within the indicated shaded time windows). This acquisition scheme is repeated for several thousand times per data point.

The x-ray signals are measured simultaneously in transmission and fluorescence yield modes (Fig. 1), using the same data acquisition strategy for all detectors, as shown in Fig. 3. The transient signal in fluorescence mode $T_{\mathrm{fl}}$ is defined via

$$
T_{f l}(E, \Delta t)=\frac{I_{p u m}^{f l}-I_{u n p}^{f l}}{I_{0}}
$$

with $I_{\text {pum }}{ }^{\mathrm{fl}}, I_{\text {unp }}{ }^{\mathrm{fl}}$ and $I_{0}$ being each baseline corrected as described above for transmission mode. Likewise, normalization of the unpumped spectra to the incident flux yields the static XAFS of the sample.

This data acquisition scheme permits us to measure the corresponding signals for every single incident $\mathrm{x}$ ray pulse, and for each data point (e.g., during a XAFS energy scan) a few thousand single $\mathrm{x}$-ray pulse intensities are analyzed in order to store the averaged value and its standard deviation into the computer.

\section{TIME-RESOLVED XAFS STUDIES}

\section{Metal-to-Ligand Charge Transfer}

As a first example we discuss the case of polypyridine complexes, here the ruthenium tris(2,2')bipyridine, $\left[\mathrm{Ru}^{\mathrm{II}}(\mathrm{bpy})_{3}\right]^{2+}$, which has been widely studied due to its potential application as an efficient light-energy converter. Absorption of a visible photon (e.g., $400 \mathrm{~nm}$ ) into the singlet metal-to-ligand-charge transfer manifold $\left({ }^{1} \mathrm{MLCT}\right)$ is followed by extremely fast (20 fs) intersystem crossing into the triplet MLCT states and subsequent energy relaxation within $[5,6]$. Finally, this charge transfer state deactivates both radiatively and nonradiatively into the ground state with a lifetime of ca. $300 \mathrm{~ns}$ at room temperature in aqueous solutions. Therefore, optical excitation leads to a change of oxidation state of the central $\mathrm{Ru}$ ion (II $\rightarrow$ III) next to a change of spin and molecular symmetry (from $D_{3}$ to $C_{2}$ ).

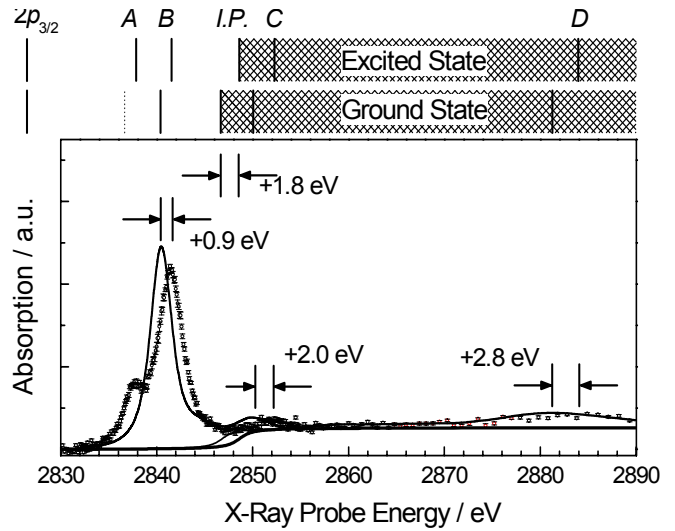

FIGURE 4. Comparison of the $L_{3}$ edge XAFS of aqueous $\left[\mathrm{Ru}{ }^{\mathrm{II}}(\mathrm{bpy})_{3}\right]^{2+}$ in its ground (thin line and points) and excited ${ }^{3}$ MLCT state (thick line and points). The edge step functions of the ionization threshold for each state is shown as well.

Figure 4 shows the $\mathrm{x}$-ray absorption spectrum around the $\mathrm{Ru} L_{3}$ edge of both the reactant complex (thin line) and the excited state $\left({ }^{3} \mathrm{MLCT}\right)$ molecule (data points with error bars). In addition, the ionization potential for both species is depicted as step functions (broadened due to the core-hole lifetime) in the figure [7]. The energetic positions of the observed features is depicted above the graph, where features $\mathrm{C}$ and $\mathrm{D}$ are above-edge resonances and the onset of EXAFS, respectively.

We observe a blue shift of all ground state features (B, C, and D), next to the appearance of a new absorption (A') below the absorption edge [8]. These arise due to the photoinduced change in oxidation state 
of the $\mathrm{Ru}$ ion, and amount ca. $1 \mathrm{eV}$ for the valence levels, and $2 \mathrm{eV}$ for the $2 s$ ionization potential (see energy bar diagram in Fig. 4) [7]. The new absorption (A') arises from the fact that the initially fully occupied $4 d t_{2 \mathrm{~g}}$ orbital (with 6 electrons) has one vacancy following phoitoexcitation, while the empty $4 d e_{\mathrm{g}}$ orbital remains empty and only shifts to higher energy. The D feature, which represents the first clear EXAFS modulation, has shifted by $3 \mathrm{eV}$, thus by more than the ionization potential itself. This additional shift is indicative of a slight decrease in the $\mathrm{Ru}-\mathrm{N}$ distance by ca. $30 \mathrm{pm}[7,9]$.

This example of $\left[\mathrm{Ru}^{\mathrm{II}}(\mathrm{bpy})_{3}\right]^{2+}$ demonstrates the rich variety of different observables, both electronic and structural, which can be retrieved from the XAFS. In the next example, we demonstrate the capability to retrieve information about the partial occupancy of valence levels in photoexcited systems.

\section{Nascent Atomic Radicals in Solution}

The optical absorption spectrum of $\mathrm{I}^{-}$in water is characterized by two broad bands (the so-called charge-transfer-to-solvent or CTTS bands) with maxima at $225 \mathrm{~nm}$ and $200 \mathrm{~nm}$. Excitation of these bands leads to abstraction of the electron from the negatively charged atom. The solvated electron is easily detected by its strong and broad near-IR (centred at $780 \mathrm{~nm}$ ) absorption. The kinetics of the latter is characterised by a multiexponential decay over fs to ns, which has been well characterized in previous studies [10-13]. Important here is that the electron population corresponds to the number of photolyzed $\mathrm{I}^{-}$ atoms. In particular, after the initial fast processes of electron-atom recombination, nearly $20 \%$ of the initial population of photolyzed species remains in the sample for hundreds of picoseconds. Based on these findings, we have probed the population of $\mathrm{I}^{0}$ species, by X-ray absorption spectroscopy at the $L_{1}$ and $L_{3}$ edges, 50 ps after electron abstraction by the pump laser.

Figure 5 shows the $L_{1}$ (initial core orbital: $2 s$ ) absorption before laser excitation, the transient difference XAS spectrum 50 ps after excitation, and the reconstructed absorption spectrum (as explained in Ref. 1) of the $\mathrm{I}^{0}$ species, based on a fractional population of the photodetached iodide of $20 \%$, which we determined from separate laser-only pump-probe experiments under similar conditions. The $\mathrm{I}^{-} \mathrm{XAS}$ is characterised by an edge around $5.2 \mathrm{keV}$, followed by marked modulations at higher energies. Being isoelectronic with $\mathrm{Xe}, \mathrm{I}^{-}$does not show bound-bound core transitions at the $L_{1}$-edge, and the modulations in the XANES region are all due to above-ionization threshold multiple scattering of the photoelectron on the solvent species [14]. Removal of the electron from $\mathrm{I}^{-}$, should lead to formation of $\mathrm{I}^{0}$ with a hole in the $5 p$ shell, that can then be accessed by excitation of the $2 s$ core orbital [15]. Therefore appearance of the $2 s \rightarrow 5 p$ resonance is a fingerprint of $\mathrm{I}^{0}$ formation. Figure $1 \mathrm{~b}$ shows the transient different absorption spectrum recorded 50 ps after laser excitation. Interestingly, it shows an increase of absorption in the pre-edge region at $\sim 5.19 \mathrm{keV}$. In addition, there is evidence of significant changes above the edge, which point to a solvent reorganisation around the solute.

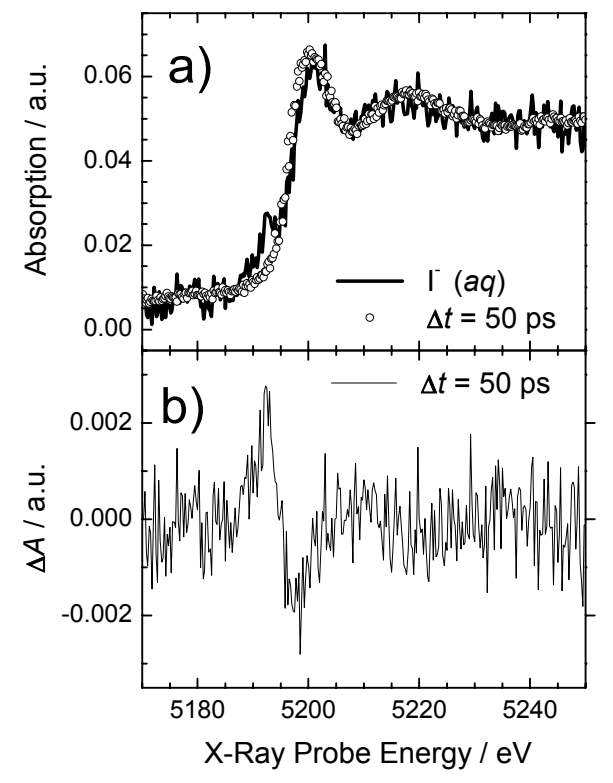

FIGURE 5. a) $L_{1}$ edge absorption spectra of aqueous $\mathrm{I}^{-}$ (open circles, $\mathrm{I}^{-}(\mathrm{aq})$ ) b) difference absorption spectrum $(\Delta \mathrm{A})$ 50 ps after laser excitation. In a), the solid line is the reconstructed $L_{1}$-edge absorption spectrum of photogenerated aqueous $\mathrm{I}^{0}$ atoms (at $\Delta \mathrm{t}=50 \mathrm{ps}$ ) using the spectra of the reactant (a, black trace) and the transient spectra (b), and taking a $20 \%$ fractional population of $\mathrm{I}^{0}$ species (see text for details).

The above results represent the first example of the observation of the changes in the solvent shell structure after light-induced electronic structure changes in an atomic solute. They could not have been retrieved by optical techniques. Although they do not probe the actual solvation process, due to time resolution, they demonstrate the power of timeresolved X-ray absorption spectroscopy as an ideal tool for the study of solvation dynamics. 


\section{Spin Crossover at Room Temperature}

Iron(II)-tris-bipyridine, $\left[\mathrm{Fe}^{\mathrm{II}}(\mathrm{bpy})_{3}\right]^{2+}$ represents a typical example of a low spin (LS) compound with the characteristic energy level scheme shown in Fig. 6. In compounds with a sufficiently low adiabatic energy difference, $\Delta \mathrm{E}_{\mathrm{HL}}^{0}$, between LS and high spin (HS) states, that is, on the order of $100-1000 \mathrm{~cm}^{-1}$, spin crossover (SCO) can be temperature-induced. In the case of $\left[\mathrm{Fe}^{\mathrm{II}}(\mathrm{bpy})_{3}\right]^{2+}, \Delta \mathrm{E}_{\mathrm{HL}}^{0} \approx 6000 \mathrm{~cm}^{-1}$ and $\mathrm{SCO}$ can only be optically triggered and stabilized to some extent at cryogenic temperatures, in the so-called Light-Induced Excited Spin State Trapping (LIESST) process [16,17]. However, in contrast to SCO compounds with low-temperature lifetimes of the light-induced state of more than $10 \mathrm{~h}$, for the LS system typical values are on the microsecond timescale. The absorption spectrum of $\left[\mathrm{Fe}^{\mathrm{II}}(\mathrm{bpy})_{3}\right]^{2+}$ is characterized by an intense broad band centred at 520 $\mathrm{nm}$ due to the singlet Metal-to-Ligand-ChargeTransfer $\left({ }^{1} \mathrm{MLCT}\right)$ state. Photoexcitation into this band is followed by a cascade of intersystem crossing steps through singlet, triplet and quintet MLCT and ligandfield (LF) states, which brings the system to the lowest-lying (HS) quintet state, ${ }^{5} \mathrm{~T}_{2}$, with almost unit quantum yield in $<1$ ps (Fig. 1) [17]. This state relaxes non-radiatively to the LS ground state within $\sim 0.6 \mathrm{~ns}$ in aqueous solutions at room temperature.

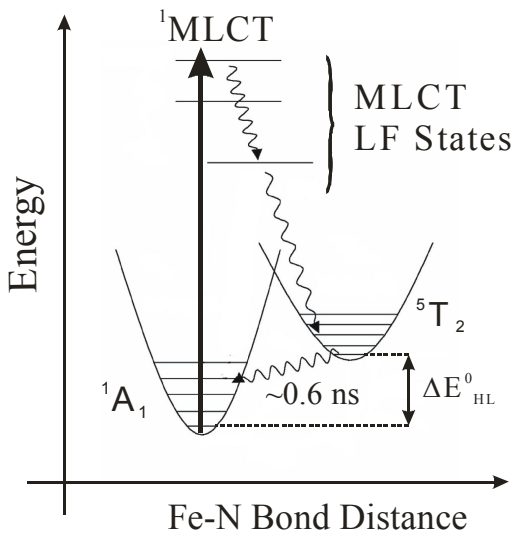

FIGURE 6. Energy-level scheme of $\mathrm{Fe}(\mathrm{bpy})_{3}$ with its photochemical cycle. A laser pulse excites the system into its metal-to-ligand charge transfer (MLCT) state, from which it cascades over MLCT and metal-centered ligand field (LF) states into the high spin (HS) ${ }^{5} \mathrm{~T}_{2}$ state. In room temperature aqueous solutions it relaxes nonradiatively back to the ground state within $0.6 \mathrm{~ns}$.

Fig. 7a shows the static XAS spectrum of the first $200 \mathrm{eV}$ above the $\mathrm{Fe} \mathrm{K}$ absorption edge of a $25 \mathrm{mM}$ aqueous solution of $\left[\mathrm{Fe}^{\mathrm{II}}(\mathrm{bpy})_{3}\right]^{2+}$. It is characterized by a number of XANES features (labeled A to D), that have already been discussed for similar Fe-compounds [18]. The features that lie $50 \mathrm{eV}$ above the edge are all electron scattering features, in particular the E-feature, which is dominated by scattering from the nearby Natoms. The exact assignment of the XANES features will not be discussed here, but all have been shown to undergo significant changes upon SCO [18-21]. This is also the case here, as can be seen in fig. $7 b$, which shows the transient difference spectrum measured 50 ps after excitation. In particular, the $\mathrm{B} / \mathrm{C}$ features gain intensity, while shifting to lower energy. The D-band intensity decreases, while the E-feature shifts to lower energies. Most of these changes are attributed to the metal-ligand bond changes. Additional changes in the high energy region (not shown here) are also clearly observed, which clearly point to a significant $\mathrm{Fe}-\mathrm{N}$ bond change. That these changes are due to the photoinduced HS complex is seen in Fig. 8, which compares the time evolution of the absorption changes at the B feature with the kinetics of ground state recovery measured by optical pump-probe spectroscopy. The latter reflects the repopulation of the LS state by the decay of the HS state and it perfectly matches the x-ray data.

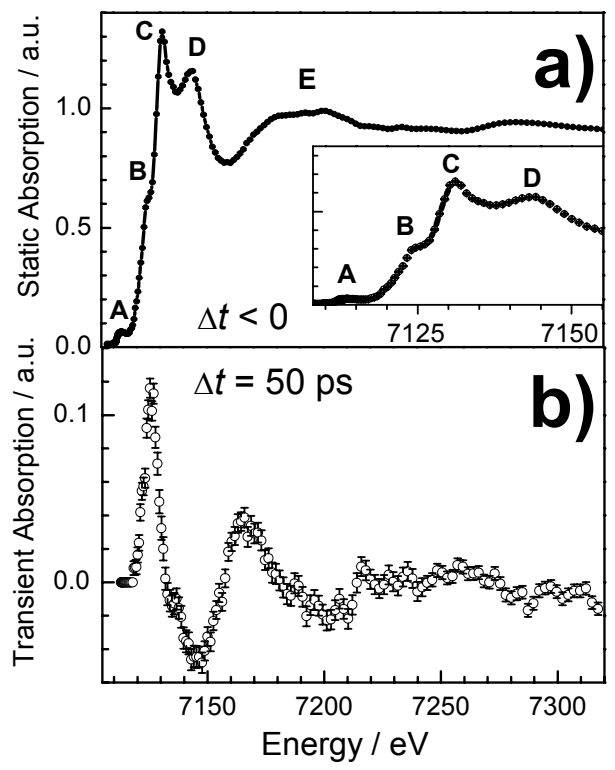

FIGURE 7. a) LS ground state XAFS spectrum of $\left[\mathrm{Fe}(\mathrm{bpy})_{3}\right]^{2+}$ (the inset zooms into the edge region) b) Transient XAFS after 50 ps.

The difference absorption spectrum (Fig. 7b) is defined as [1]

$T(\mathrm{E}, t)=f(t) \cdot\left[A_{\mathrm{HS}}(E, t)-A_{\mathrm{LS}}(E)\right]$ 
where $f(\mathrm{t})$ is the fractional population of the HS complex at time $t(50 \mathrm{ps}$ in fig. $2 \mathrm{~b}), A_{\mathrm{LS}}(E)$ is the absorption spectrum of the LS complex (Fig. 7a), and $\mathrm{A}_{\mathrm{HS}}(\mathrm{E}, t)$ that of the HS complex, at the time $t$ following the photoexcitation. In order to extract the excited state structure correctly, $f(\mathrm{t})$ must be known, and we measured a value of $22(2) \%$ at $t=50 \mathrm{ps}$, in laser-only pump-probe experiments carried out under identical experimental conditions as in the present measurements.

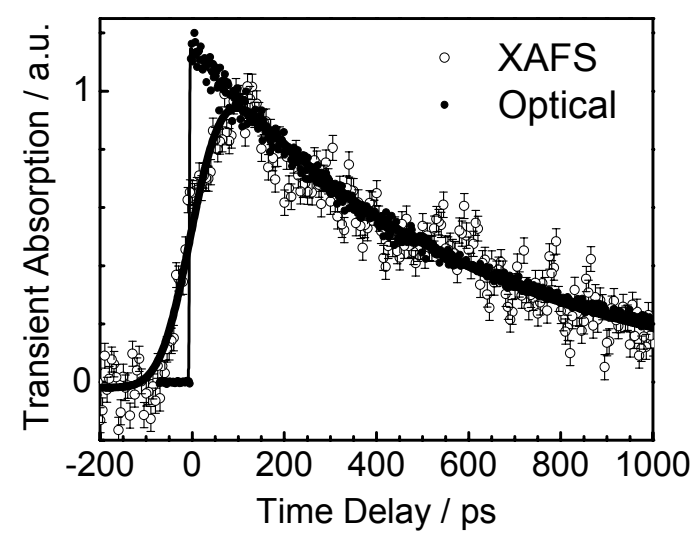

FIGURE 8. Kinetics of the difference XAS signal of aqueous $\mathrm{Fe}$ (bpy) 3 at room temperature, recorded at $7126 \mathrm{eV}$ (open circles) upon $400 \mathrm{~nm}$ excitation, and that of the optical signal (triangles) recorded in transmission at $523 \mathrm{~nm}$, and reflecting the repopulation of the ground state together with fit curves. The solid lines represent the fit curves, which in the case of the X-ray signal is a monoexponential convoluted with the $100 \mathrm{ps}$ width of the X-ray pulse.

We have used these values for a simulation of the transient EXAFS spectrum shown in Fig. 9. In Fig. 9a the static EXAFS has been compared with the fitted outcome of the EXAFS calculation performed with the FEFF 8.20 code over a large energy range (ca. $650 \mathrm{eV}$ above the edge). The results yield an excellent agreement with the LS structure as determined by static x-ray crystallography. The fit was done by taking into account all scattering contributions and by varying their Debye-Waller factors to best match the experimental data. In addition, we have refined the $\mathrm{Fe}$ $\mathrm{N}$ bond distance by setting it as a free running parameter and the obtained result agrees very well with the published value of $1.97 \AA$ within its uncertainty of $0.01 \AA$.

In the next step, we have performed several EXAFS calculations for several structural models with different $\mathrm{Fe}-\mathrm{N}$ distances (the actual models were obtained by moving all 3 bpy ligands symmetrically outwards the $\mathrm{Fe}$ atom and thus modifying the bond lengths of all constituent atoms with respect to the absorber) in steps ranging from 0.03 to $0.005 \AA$. We then calculated the transient EXAFS using $f=22 \%$, and the obtained ground state fit result from Fig. 9a. An excellent agreement is obtained for $\Delta R_{\mathrm{Fe}-\mathrm{N}}=0.2$ (0.02) $\AA$, and the resulting transient simulation is depicted in Fig. $9 \mathrm{~b}$ together with the data.
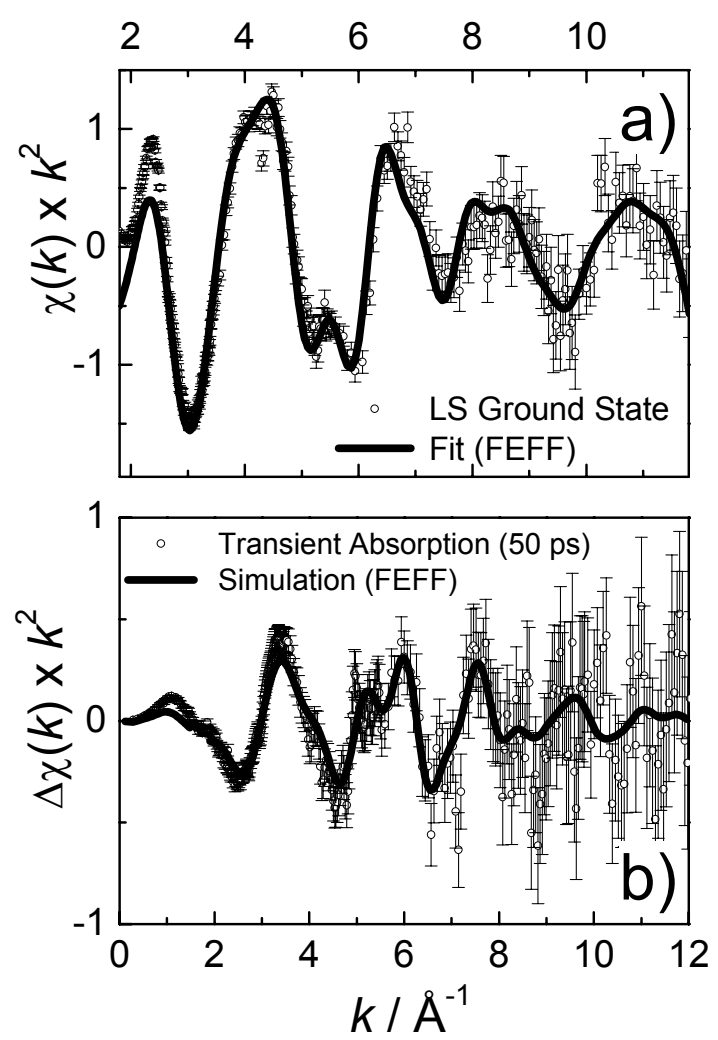

FIGURE 9. a) LS state EXAFS spectrum together with the fit curve yielding $\left.R_{\mathrm{Fe}-\mathrm{N}}=1.97(0.01) \AA . \mathrm{b}\right)$ Transient EXAFS after 50 ps together with a transient simulation for $\Delta R_{\mathrm{Fe}-\mathrm{N}}=$ $0.2(0.02) \AA$.

We are currently refining the fit procedure to reliably quantify the actual bond length increase, as measured in the transient absorption spectra. This novel approach may yield uncertainties on the order of conventional state-of-the-art EXAFS studies, i.e., in the 0.01-0.001 $\AA$ range. This is because transient structural changes are rather small compared to the ground state structure. With the precise knowledge of the ground state structure (e.g., from crystallographic measurements), the fit procedure for determining the excited state becomes less ambiguous. Using the transient XAFS instead of the excited state XAFS spectrum can then deliver much more precise values for the structural changes. Currently, a bond length increase of $0.2(0.02) \AA$ seems reasonable both from the comparison of the transient spectra as well as from 
theoretical calculations. A full multiple scattering analysis of the photoinduced changes in the XANES and of the EXAFS region is currently underway, which should deliver a precise value for the Fe-N bond distance change.

The physical time scale for relaxation from the singlet ${ }^{1}$ MLCT to the HS state is expected to be $<1 \mathrm{ps,}$ occuring via several intermediate singlet and triplet states, which are each accompanied by a structural modification of the $\mathrm{Fe}-\mathrm{N}$ bond distances. Optical spectroscopy has so far not been able to resolve these steps, and is unable to determine their structures. Future extension of these experiments using femtosecond x-ray pulses may soon provide better insight into this relaxation process.

\section{CONCLUSIONS}

This work shows examples of picosecond x-ray absorption spectroscopy applied to chemical systems in liquids. Time-resolved XAFS provides detailed information about the electronic state of short-lived reaction intermediates. Already knowledge of the oxidation state can aid in understanding the whereabouts of the photoexcited electron, as shown for the case of $\mathrm{Ru}(\mathrm{bpy})_{3}$, but also about possible fractional (re)population of valence states, as shown for the nascent iodine atoms in water. But XAFS methods are capable of simultaneously monitoring the geometric structure with high spatial resolution, as illustrated for the high-spin state of $\mathrm{Fe}(\mathrm{bpy})_{3}$.

These properties make this new tool suitable to study various phenomena in rather complex systems in disordered media. Thus it appears now feasible to study the very first instants of biological activity of proteins in physiological media. Ultrafast $\mathrm{x}$-ray absorption spectroscopy holds a high potential to embark into structural dynamics in condensed phase systems. With the new Femtosecond x-ray sources becoming available these days, it is also possible to study nuclear wave packet dynamics exploiting XAFS tools.

\section{ACKNOWLEDGMENTS}

This work is funded by the Swiss National Science Foundation (FNRS), via contracts 620-066145, 200021-107956 and 200021-105239 and by the Swiss Light Source (SLS). We thank the microXAS beamline staff, M. Willimann, M. Harfouche and B. Meyer, for their assistance and help during the measurements.

\section{REFERENCES}

1. C. Bressler and M. Chergui, Chem. Rev. 104, 1781 (2004).

2. M. Saes, W. Gawelda, R. Abela, D. Grolimund, F. van Mourik, S. L. Johnson, P. A. Heimann, R. W. Falcone, R. W. Schoenlein, A. M. Lindenberg, M. Chergui, C. Bressler, Rev. Sci. Instrum. A 75 (1), 24-30 (2004).

3. W. Gawelda, C. Bressler, M. Saes, et al., Physica Scripta T115, 102 (2005).

4. W. Gawelda, V.T. Pham, A. El Nahhas, M. Kaiser, Y. Zaushitsyn, S. L. Johnson, D. Grolimund, R. Abela, A. Hauser, C. Bressler, M. Chergui, AIP Conf. Proc. 882, 31 (2007).

5. A. N. Tarnovsky, W. Gawelda, M. Johnson, C. Bressler, M. Chergui, J. Phys. Chem. B 110, 26497 (2006).

6. A. Cannizzo, F. Van Mourik, W. Gawelda, G. Zgrablic, C. Bressler, M. Chergui, Angew. Chem Intl. Ed.. 45, 3174 (2006).

7. W. Gawelda, M. Johnson, F. M. F. de Groot, R. Abela, C. Bressler, and M. Chergui, J. Am. Chem. Soc. 128, 5001 (2006)

8. M. Saes, C. Bressler, R. Abela, D.Grolimund, S. L. Johnson, P. A. Heimann, M. Phys. Rev. Lett. 90, 474031 (2003)

9. M. Benfatto, S. Della Longa, K. Hatada, K. Hayakawa, W. Gawelda, C. Bressler, M. Chergui, J. Phys. Chem. B 110, 14035 (2006)

10. J. A.Kloepfer, V. H.Vilchiz, V. A.Lenchenkov, A. C.Germaine, S. E. Bradforth, J. Chem. Phys. 113, 6288. (2000)

11. J. A. Kloepfer, V. H. Vilchiz, V. A. Lenchenkov, X. Y. Chen, S. E. Bradforth, Journal of Chemical Physics 117, 766 (2002)

12. M. C. Sauer, I. A. ShkrobR. Lian, R. A. Crowell, D. M. BartelsX. Y. Chen, D. Suffern, S. E. Bradforth, Journal of Physical Chemistry A 108, 10414 (2004)

13. H. Iglev, A. Trifonov, A. Thaller, I. Buchvarov, T. Fiebig, A. Laubereau, Chemical Physics Letters 403, 198 (2005)

14. H. Tanida, K. Kato, I. Watanabe, Bulletin of the Chemical Society of Japan 76, 1735 ( 2003)

15. C. Bressler, M. Saes, M. Chergui, D. Grolimund, R. Abela, P. Pattison, Journal of Chemical Physics 116, 2955 (2002)

16. A. Hauser, Topics in Current Chemistry 234, 155 (2004).

17. C. Brady, J. J. McGarvey, J. K. McCusker, et al., Topics in Current Chemistry 235, 1 (2004).

18. C. Hannay, M. J. HubinFranskin, F. Grandjean, et al., Inorganic Chemistry 36, 5580 (1997).

19. a) H. Oyanagi, T. Tayagaki, and K. Tanaka, Journal of Physics and Chemistry of Solids 65, 1485 (2004), b) H. Oyanagi, T. Tayagaki, and K. Tanaka, Journal of Luminescence 119, 361 (2006).

20. a) J. Kusz, H. Spiering, P. Gütlich, J. Appl Cryst. 33, 201 (2000), b) J. Kusz, D. Schollmexer, H. Spiering, P. Gütlich, J. Appl. Cryst. 38, 528 (2005).

21. M. L. Boillot, J. Zarembowitch, J. P. Itie, et al., New Journal of Chemistry 26, 313 (2002). 